\title{
Un marco teórico para interpretar el efecto instituyente de los estímulos económicos al profesorado universitario: datos de la experiencia mexicana
}

\author{
Rosalía Susana Lastra Barrios, Universidad de Guanajuato, México \\ Oscar Comas Rodríguez, Universidad Autónoma Metropolitana, México
}

\begin{abstract}
Resumen: Un reto por atender en las ciencias sociales y humanas es dar orden a la dispersión conceptual y metodológica que conduzca a establecer una teoría del valor de la producción académica. Lo presente abona en tal dirección, aportando un marco teórico-interpretativo que exige un intenso trabajo interdisciplinar, dispuesto para conocer de forma más sistemática el fenómeno que propicia la aplicación de los oficialistas programas de estímulos económicos (PEE) mexicanos a la labor del profesorado universitario, asi como sus efectos y tensiones instituyentes consecuentes. Se escudriña desde el significado económico hasta la significancia sociológica, a partir de la información obtenida de la muestra colectada entre integrantes del Sistema Nacional de Investigadores. Se finaliza infiriendo sobre lo urgente de renovar algunos aspectos axiales.
\end{abstract}

Palabras clave: estímulos económicos, profesor universitario, institucionalismo sociológico

\begin{abstract}
A challenge in the social sciences and humanities is to create an order in the conceptual and methodological dispersion which will enable us to establish a value theory for academic production. This article contributes to this effort by providing a theoretical-interpretative framework that requires intensive interdisciplinary work. It is designed to allow us to more systematically understand the effect of the Mexican economic stimulus programs on the work of university professors as well as its concomitant institutional tensions. The phenomenon is examined in a variety of different ways, from an economic perspective a sociological one, using the information obtained from a sample collected from the National Researcher System. The conclusion is about the urgency of renewing certain pivotal aspects.
\end{abstract}

Keywords: Economic Stimuli, University, Faculty Members, Theoretical Framework

\section{Antecedentes}

$\mathrm{C}$ onocido que en la base del comportamiento social están las motivaciones, para entenderlas, es necesario adentrarse, entre otras cosas, en el análisis del contenido de las instituciones prevalecientes en cada campo organizacional, y así aproximar las claves sobre aquello que impulsa las decisiones y el accionar. En México, desde mediados de la década de los años 80, el poder ejecutivo, a través primero del Consejo Nacional para la Ciencia y la Tecnología (CONACYT) y luego de la Secretaría de Educación Pública (SEP), decretaron la creación de los $\mathrm{PEE}^{1}$ para alentar el desempeño del profesorado universitario. Empero, su aplicación en las organizaciones de educación superior actúa interviniendo los lineamientos de la dinámica institucional y del conocimiento de formas no siempre coordinadas, lo cual origina efectos contradictorios en las formas de entender lo que ha de ser el papel del "académico ideal".

\footnotetext{
${ }^{1}$ En el CONACYT, el Sistema Nacional de Investigadores (SNI), en 1984 y en la Subsecretaría de Educación Superior, el Programa de Estímulo al Desempeño del Personal Docente (ESDEPED), en 1991 y el Programa para el Mejoramiento del Profesorado (PROMEP), en 1996, entre los principales, además de los locales.
}

Revista Internacional de Ciencias Sociales

Volumen 2, Número 2, <http://lascienciassociales.com>, ISSN 2530-4909

(C) Global Knowledge Academics, R. S. Lastra Barrios, O. Comas Rodríguez.

Todos los derechos reservados. Permisos: soporte@gkacademics.com

Republicado de Revista Internacional de Ciencias Sociales Interdisciplinares 2(2), 2013 (pp. 93-103) 
Por tanto, la pregunta guía es ¿cómo dar seguimiento a los efectos instituyentes de los PEE a partir del enfoque de los académicos receptores de la acción?

\section{Términos de referencia}

No se conocen marcos teórico-interpretativos creados al efecto, por lo cual aquí se asume tal responsabilidad. La explicación de la traza general de la presente propuesta teórico-metodológica requiere de revisar los conceptos a utilizar para aproximarnos de forma ordenada al conocimiento del fenómeno propuesto. Lo primero es no tomar como sinónimos los términos institución y organización.

Por institución se entiende un determinado sistema de reglas con sus estructuras de significado, adquirido de forma casi siempre inconsciente y practicado por amplios conglomerados humanos. Operativamente, refiere un patrón de actividad supra-organizacional, constituido por símbolos y prácticas materiales, donde individuos y organizaciones producen, conducen y reproducen su sustancia, dando significado a la experiencia para utilizar el tiempo y el espacio (Meyer y Rowan, 1992). La importancia de estudiar las instituciones es que permite su reconocimiento como fuerzas contextuales y mecanismos dimensionales que configuran la realidad organizacional, así como el impacto de ésta hacia aquellas (Zucker, 1991), en una especie de traducción de argumentos macro-institucionales en cadenas de micro-actuaciones.

La existencia etérea de las instituciones, contrasta con la existencia delimitada de la organización. Por organización se entiende la configuración del fenómeno social expresable en un cuadro de elementos que estructuran objetivos de un grupo humano con filiación, definible en tiempo y espacio, donde perviven individuos realizando acciones formales e informales, en función de roles, intereses, decisiones y expectativas, que a veces les identifica y otras les contrapone, dados sus respectivos marcos institucionales que escenifican fenómenos de tensión-distensión.

El acontecer de las prácticas institucionales en las organizaciones configura un determinado Patrón Institucional, el cual queda definido como el proceso establecido por reglas, guiones y clasificaciones que se dan por hecho (Powell y Di Maggio 1991: 50) y que constituyen, por tanto, una forma reiterativa para persistir: lo común es que tal Patrón devenga en una conjunción de estereotipos sociales, con cierto proceso de reproducción relativamente auto-activado (1991). El Patrón Institucional latinoamericano queda configurado por las prácticas hibridas sui géneris resultantes de las instituciones mercado capitalista, Estado burocrático, democracia y catolicismo.

Lo que se persigue con el método que se presenta a continuación, es establecer para un determinado grupo organizacional universitario, los efectos que explican la cercanía o distancia del Patrón Institucional con el Patrón en Institucionalización, éste definido como la identificación del sistema de reglas, guiones y clasificaciones en introyección entre los integrantes.

Se acepta la regla de contraste de que “... cuando los alejamientos del Patrón [institucional] son contrarrestados de manera regular por controles construidos socialmente y activados de manera repetitiva (por castigos o recompensas) podemos hablar de un patrón institucionalizado" (Romero, 2001: 14), control que se asume, es realizado por los PEE.

La noción que mejor apoya el acercamiento al vínculo entre la introyección de los lineamientos de los PEE con el medio universitario, es la de mito racionalizado (Meyer y Rowan, 1992). Mito, en tanto constituye una creencia ampliamente sostenida dese alguna autoridad, pero de difícil verificación objetiva, siendo cierta, en la medida que es creída -como es el caso de que el estímulo económico activa la generación de conocimiento-, y racionalizado en tanto que quien origina el mito le da la forma de regla que especifica comportamientos necesarios para alcanzar un fin considerado genuino -como que participando en los PEE se logra ser un "académico competitivo" y, entonces, forjar una mejor universidad.

Este tipo de mito aporta legitimidad social al garantizar el consenso necesario que, en el límite, hace que las universidades públicas justifiquen su pertinencia o subsistencia, cambiando, de ser necesario, su estructura formal, dados los requerimientos de aceptación de criterios exter- 
nos. Sin incurrir en paranoia, el objetivo de quien crea los mitos suele ser legitimar -interna y/o externamente- un desempeño, haciendo aparecer la aplicación de sus reglas como efectivas, aún sin comprobación a la luz de los objetivos formales.

Una consecuencia de los mitos en que se basan las lógicas de instituciones disímbolas, como las del Patrón institucional mexicano, suele ser el surgimiento de contradicciones que dificultan el funcionamiento de ciertas estructuras universitarias y sus procesos derivados; es el caso de cuando la lógica institucional del mercado capitalista sale de la esfera de la eficiencia económica para penetrar en la lógica de la actividad académica, incrementando su participación en la explicación de, por ejemplo, el deber ser de la forma de activar el conocimiento o acciones justificativas por el establecimiento de algún tipo de democracia.

Friedland y Alford teorizan que cuando la contradicción interinstitucional se vuelve conflicto abierto, se tiene una fuente de cambio y resistencia, pudiendo los implicados movilizarse para defender los símbolos y las prácticas de una institución de las consecuencias del cambio en otras, o intentar exportar/importar los símbolos o prácticas de una, con el fin de transformar a otra a la propia conveniencia (Friedland y Alford, 1991).

El caso cabe cuando un académico apoya las fórmulas neoliberales para ser remunerado y, al mismo tiempo, está de acuerdo en que sea el sector público quien fije los criterios -no los usuarios de su servicio-, clamando además que las decisiones de evaluación se tomen por prácticas democráticas en su claustro académico. La contradicción puede ser aparente, requiriéndose acopiar más información que viabilice reconstruir las racionalidades institucionales subyacentes.

A juzgar por ciertas representaciones previamente sondeadas, la situación alcanza a tocar la identidad de los académicos que expresan cómo se ven ante el desafío de proseguir la senda dictada por su consciencia o asumir los nuevos retos planteados en su campo organizacional, adicionales a las complejas tensiones de su quehacer, caracterizadas por:

...cambio rápido en los saberes de la enseñanza, disminución del compromiso social..., obsesión por la técnica, lo privado como sustituto de lo público, la primacía de los valores económicos, la competitividad como rectora de la profesión y la incorporación por moda de paradigmas pedagógicos que ofrecen eficacia ante el reclamo de una educación pertinente" (López, R. 2003: 245).

Las contradicciones reales del patrón institucional se traducen en que el académico tiene que construir conductas y discursos congruentes con las prácticas que de él se esperan, respondiendo al contexto de la visión de sociedad del conocimiento que percibe y al papel de universidad que prefigura.

Nótese que se elude la utilización de los conceptos "producto" y "calidad", tomando distancia a lo que significan cuando se aplican acríticamente al trabajo académico.

\section{Los PEE, instrumento instituyente de prácticas académicas inéditas}

Los PEE en México nacen bajo el cobijo público, posterior a lo acontecido con sus antecesores, los llamados programas de Merit Pay, en países con sistemas universitarios más estructurados, pero que los abandonaron desde hace más de medio siglo, por los efectos contrarios o inocuos al objetivo de dinamizar el conocimiento científico (Comas, 2003); contar con académicos de estirpe y salarios adecuados fue determinante para tal abandono.

Un primer mito institucional con el que nacen, es que aunque se suele creer que en México avanza la institucionalidad del capitalismo - contraria a la tradición socialista para la educación-, el establecimiento de estructuras por mercado de puntos no alcanza para sostener tal afirmación, pues las actividades académicas son calificadas por comités de pares académicos y falta el ingrediente esencial, que es el escrutinio del usuario directo del servicio.

Otro mito emerge del origen de los PEE. No fueron creados bajo la mística de potenciar la generación y mejor distribución del conocimiento; lo fueron para repartir de forma rápida y, por tanto, objetivista, un recurso extraordinario para acallar el clamor de los académicos por salarios en continuo deterioro, asumiendo que también servirían para remover prácticas conformistas. 
La perpetuación de la estructura evaluativa así surgida, hoy se apoya en el axioma de que el mismo orden organizativo sirve para zanjar inconformidades salariales (necesidad exterior) y estimular la dinámica del trabajo académico (necesidad interior), requiriéndose indagar las disrupciones identitarias provocadas por la yuxtaposición de ambos órdenes, aunque los promotores de los PEE insisten en sostener el mito de que uno objetivo conduce sin problemas al otro.

El diseño de los llamados sistemas de estímulo cuenta con la legitimidad que da la intervención de connotados académicos, que al inicio procuraron especializarse en alguna función académica, pero que hoy tienden a requerir la multifuncionalidad para prefigurar el "académico ideal", perfil que no acaba por convencer, sea por la rigidez que impone la configuración real vocacional de cada individuo, así como por no ser una fórmula que ayude a reconfigurar la misión especial de cada universidad para incrementar la atención a las necesidades sociales de cada entorno. De aquí la necesidad de indagar entre los académicos con qué perfil funcional se identifican e inferir distancias con lo promovido.

Claro que la repartición de recursos -que para quienes participan en más de un PEE llega rebasar el doble de su salario base-, ha producido efectos benéficos, como la procura de más grados académicos, publicaciones, comunicarse en congresos, incorporarse a cuerpos académicos, entre los principales. Empero, en el largo plazo, la costumbre a este tipo de estímulo termina por exacerbar las reglas, impulsando efectos instituyentes que conducen a la segregación grupal y a sofisticadas formas de adulteración de las actividades de la labor, con poca opción a detenerse en consideraciones éticas.

La conseja de que la evaluación tiende a pervertirse cuando ciertos desempeños se sujetan al logro de recompensas económicas, parece cumplirse, pues la esencia académica es equiparable al desfogue que siente un artista al crear su obra, siendo una facultad no mejorable al sujetarse a formas de coacción externa.

Otro mito conlleva a indagar lo que se piensa de la estructura de valores a las actividades académicas. Llevar al límite la exigencia del apilamiento de constancias de actividades emitidas por diversos tipos de autoridades, con más valor en las investigativas, explica la reducción del esmero en el aula, en la innovación científica y el rigor con que se participa en los eventos académicos, en ocasiones con la "permisividad" de los evaluadores.

Así, cabe explorar la opinión del gremio sobre la modelación de criterios y su complicación con la dimensión "poder", por la forma en que llegan a ser aplicados por los comités evaluadores federales, que aunque yuxtapuestos para verificar los dictámenes, son incuestionables a pesar de ser "juez y parte" de un sistema que deja la verificación de la valía de cada expediente a expertos disciplinares, pero sin profesionalización en la evaluación de desempeños, puerta abierta al control estamental por intereses de grupo.

La traza general anterior, de las principales circunstancias implicadas en el fenómeno, sirven para contextualizar la forma en que se fueron fundamentando e interpretando las evidencias empíricas ejemplificadas en la sección 5 .

Sabido que en muchos países se discute la aplicación de modalidades similares de éste fenómeno evaluativo-estimulativo, la alerta va por tomar providencias para mejorar la experiencia. No se afirma que lo que se abandonó en unas latitudes no puede ser útil en otras o en otro tiempo, sino que se requiere estar preparados metodológicamente para aprehender el fenómeno. Una medida es crear marcos interpretativos para la meta-evaluación, que ayude a dar cuenta del seguimiento de los efectos instituyentes entre los receptores de la acción.

\section{Marco teórico-metodológico}

El "artefacto" investigativo aquí propuesto, producto de la discusión teórica que es posible consultar en (Lastra, 2011 y Lastra y Comas 2011), comulga con el ideario del Nuevo Institucionalismo Sociológico (March y Olsen, 1989 y Powell y Di Maggio, 1991), debido a la convergencia con el propósito de conocer la incidencia de los procesos institucionalizados en la dinámica organi- 
zacional y sus ocupantes, prestando atención a las dimensiones poder, historia, marco valorativo y, sobre todo, al voluntarismo de las personas y su capacidad para construir el mundo en el que conviven, utilizando la detección del seguimiento de reglas por las cuales se decide o se desea persistir o cambiar (Zucker, 1991 y Coronilla, R. 2004), en lo individual o el desempeño de la labor.

A partir de la revisión literaria sobre el tema (Comas y Lastra, 2012), se estableció un mapeo del fenómeno, resolviéndose ordenarlo en cuatro corpus de incidencia por los cuales, se sostiene, es posible observar la senda por la que transitan los mitos racionalizados transportados por los PEE hasta albergarse en los sujetos de la acción; ellos son: 1) el contexto internacional, 2) el ámbito de la educación superior, 3) los mitos institucionalizados existentes en cada universidad 4) lo instituido en el profesorado. Entre cada corpus, se prefigura el espacio analítico, denominado intersticio, propicio para identificar y reflexionar sobre la verticalidad o inflexiones con la cual pasa la regla institucional de un corpus a otro.

La continuidad intersticial de la regla instituyente en análisis se interpreta de tal forma que: a mayor alineamiento podrá decirse que el grado de institucionalización es más armónico, aunque no por ello, exento de contradicciones a ser detectadas. A mayor número de quiebres en el tránsito de la regla de que se trate (contradicciones académicos-PEE), se inferirá mayor tensión organizacional del académico y/o el grupo hacia el ambiente universitario promovido por los PEE.

Con lo escudriñado en los primeros dos corpus se determinaron las nociones predominantes en el medio universitario mexicano, traducidas en las prescripciones de los PEE. Se les da el tratamiento de mitos (Meyer y Rowan, 1992), al no desear de momento debatir su cumplimiento real.

La siguiente ilustración facilita el entendimiento de la estructura del marco interpretativo:

口

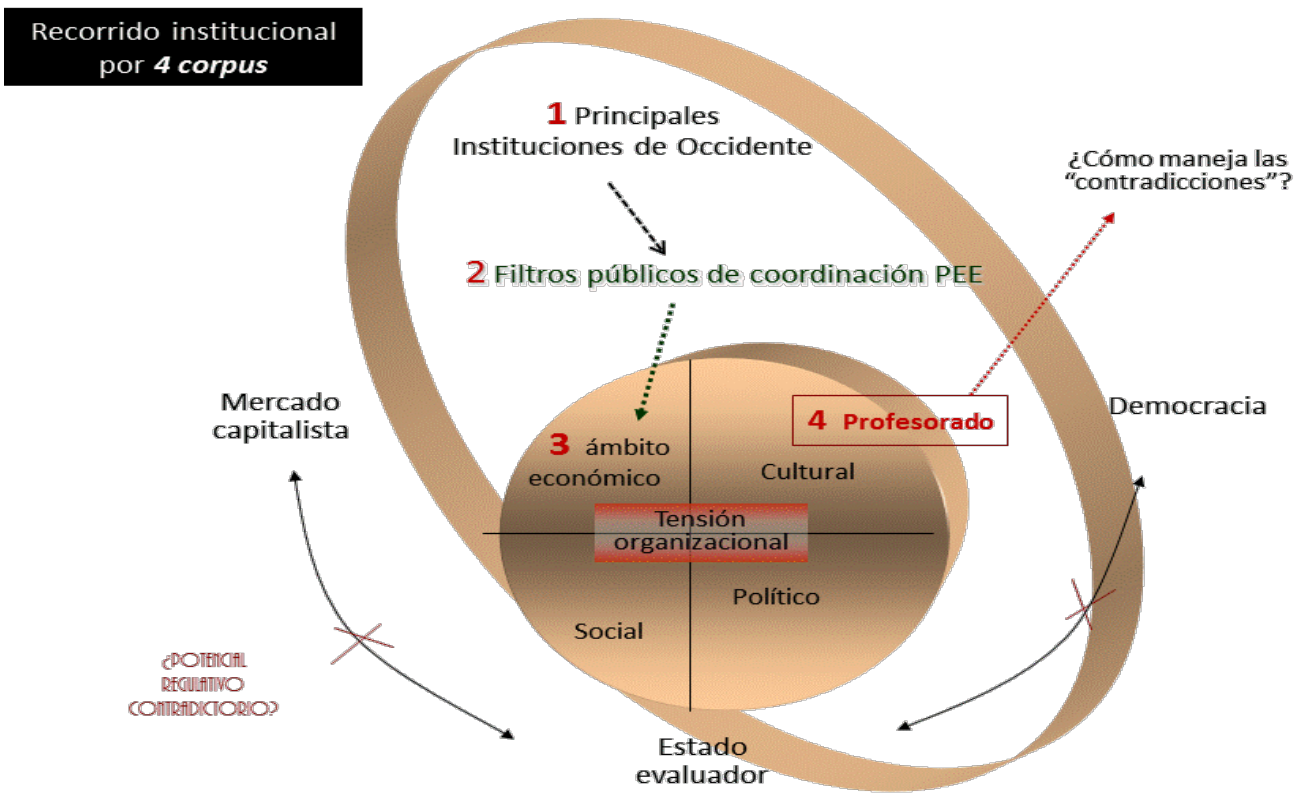

Fuente: Elaboración propia

La pregnancia de la institución mercado capitalista se sondeó formulando preguntas sobre lo que se ve que ocurre o se preferiría que ocurriera con: la empresarialización de la universidad 
pública -y por tanto con la labor académica-, la necesidad de participar en lo que se entienda por globalización, el desempeño multifuncional, el sentido de la modernización universitaria, la justa retribución por puntos y el sentido ético; la inclinación a la institución Estado evaluador se indagó a través de los mitos: ajuste en los grados de autonomía universitaria, pertinencia de la política pública para la educación superior, así como la del sistema credencialista, lo oportuno de la planeación a plazos y la legitimidad y legalidad de la forma de evaluar y estimular. Finalmente, respecto a la introyección de la institución democracia, dispuesta a tramos en los procesos de evaluación de los PEE, se utilizaron los mitos sobre la mejora en el flujo de la información, las transparencia en las decisiones, el desempeño de los pares evaluadores, la adecuación del sistema de estímulos y la comunicación.

Debido a que los procesos de institucionalización se prueban en conglomerados humanos, fue directa la decisión de utilizar el cuestionario como primera instancia para la recogida de información. Sin limitar el número de preguntas, resultaron 80, de las cuales 30 corresponden a datos personales generales con los cuales se caracteriza al grupo.

Los rangos de respuestas se diseñaron en forma no convencional, siempre con final abierto, para quienes desean aportar más información. Se decidió la redacción retadora por tratarse de académicos acostumbrados al diseño estándar de respuestas, evitando que acondiciones el sentido.

Se decidió iniciar la aplicación entre los 16,650 integrantes del Sistema Nacional de Investigadores (SNI 2010) de todo el país, en sus 5 niveles y 515 organizaciones, por ser el grupo que ofrece mayor probabilidad de conocimiento de los PEE, aunque pudo ser cualquier otro.

La solicitación a participar se realizó por correo electrónico. El envío se eligió al inicio de enero, por ser fecha lejana a las presiones laborales y a la presentación de reportes de trabajo. No todos recibieron el correo por problemas técnicos (solo $3 / 4$ partes), pero de los que sí lo recibieron y atendieron, la muestra por disponibilidad quedó configurada por un $9.3 \%$ del total de integrantes del SNI, lo que significa que la interpretación de respuestas se basa en 1,549 cuestionarios (aunque se pudiera cuestionar la representatividad estadística, igual se trabaja con lo disponible, aunque con menos confianza de representatividad). La captura y elaboración estadística de la información se realizó automatizadamente en la base de datos alemana LIME, para encuestas (Lastra, 2011).

\section{Convite de disciplinas}

El logro de acercamientos más certeros en el análisis institucionalista exige del esfuerzo interpretativo que transita de la visión economicista (estudio de la formación racional de las elecciones), a la sociológica (estudio de las bases no racionales que impiden elegir) llegando a la institucional (estudio de la oposición entre órdenes trans racionales, es decir, más allá del juicio de lo racional). Aunque la idea de estructurar este marco interpretativo fue de un economista, fue secundada por un par de organizacionólogos humanistas y luego tres investigadores educativos enriquecieron la visión de la configuración del objeto de estudio, los mitos racionalizados y el diseño de las preguntas del cuestionario.

El diseño de las preguntas giró con la intervención de un filósofo y un metodólogo, afinándose con la vista previa de un historiador de la educación superior, también útil para delimitar los corpus e intersticios.

Por los mitos explorados se deduce la relevancia de contar con la mirada interpretativa de los sociólogos, epistemólogos, politólogos y psicólogos sociales. No contar con al menos tales enfoques, compromete la credibilidad de la mirada única, incrementando indeseablemente, en consecuencia, el sesgo interpretativo sobre la institucionalización en marcha en el grupo en estudio; las miradas parciales permiten inferencias menos significativas sobre la realidad instituida. La traducción de lo anterior en la encuesta no puede prescindir del sagaz apoyo de un ingeniero en sistemas, de preferencia con alguna formación humanista. 
Las opiniones de los distintos especialistas no tienen que ser simultáneas. La secuenciación intersticial orienta la pertinencia analítica de cada intervención. Con el interés común de conocer los rasgos que caracterizan el ambiente institucional en el que los profesores recrean la labor, se amplía la posibilidad de encontrar las formas culturales teóricamente estandarizables buscadas, de tal forma que se avance en el establecimiento de los principios de alguna teoría del valor otorgado a la producción académica desde sus sujetos.

\section{Colecta de información e interpretación primaria}

E1 73\% de las respuestas se recibieron durante los primeros 4 días y el resto se diluyó durante los días siguientes del mes, cuando se cerró la recepción. Sólo 63 académicos contactaron por correo para clarificar el sentido de alguna pregunta o para indagar más sobre el uso que se daría a la información. En las preguntas sobre la institución Estado evaluador (segundo tercio del cuestionario), se identifica tendencia al abandono más rápido del cuestionario, habiéndolo completado sólo la mitad de quienes lo iniciaron, por parecerles extenso o con preguntas que no desearon responder.

El inicio del ordenamiento informativo es con la caracterización de los rasgos generales del grupo, pero aquí no se presentará al no ser necesaria para mostrar la utilidad potencial del método propuesto. Sería distinto si se tratara de un estudio de caso. De momento, basta saber que la base de datos permite cruzar los rasgos como edad, género, área del conocimiento, etc., con las respuestas a cada noción institucional.

La siguiente tarea es el agrupamiento de tendencias en cada pregunta de las nociones institucionales exploradas, la cual se realiza con la lectura horizontal. Dado el rango extrapolado, es posible clasificar las respuestas del grupo según las claves: coincidente $(\mathrm{C})$, si la respuesta mayoritaria cayó entre el $60-100 \%$; no coincidente (NC) en el rango $0-40 \%$, o con coincidencia media (CM) en el rango $40-60 \%$.

Un sencillo ejemplo del paso de la cuantificación horizontal de respuestas a la cualificación interpretativa vertical de tales agrupamientos es el que resulta de la exploración de lo instituido en torno a la noción multifuncionalidad, interesante además porque permite mostrar cómo, tratándose de un mismo aspecto, se detecta tensión entre lo que asume la mayoría del grupo para su labor, su lugar de trabajo o para la universidad pública. Se tiene la siguiente información:

\begin{tabular}{|c|c|}
\hline $\begin{array}{l}\text { Percepción para el } \\
\text { logro del perfil multi- } \\
\text { funcional }\end{array}$ & $\begin{array}{l}\text { Pregunta III-10. El perfil multifuncional me es alcanzable igual que: A todos } \\
13 \% \text {, La mayoría } 22 \% \text {, Los que se esfuerzan } 31 \% \text {, Una élite } 8 \% \text {, Otro } 5 \% \text {, } \\
\text { Sin respuesta } 21 \% \text {. Indicador: C }(13 \%+22 \%+31 \%=66 \%) \text {. }\end{array}$ \\
\hline $\begin{array}{c}\text { Preferencia por ser } \\
\text { profesor multifuncional }\end{array}$ & $\begin{array}{l}\text { Pregunta III-13. Prefiero ser profesor: Especializado (docencia, investigación } \\
\text { u otro), } 62 \% \text {; Multifuncional, } 22 \% \text {; Me da igual, } 2 \% \text {; Otro, } 2 \% \text {; Sin respuesta, } \\
11 \% \text {. Indicador: C. }\end{array}$ \\
\hline $\begin{array}{l}\text { Adecuación de la multi- } \\
\text { funcionalidad para el } \\
\text { lugar de trabajo }\end{array}$ & $\begin{array}{l}\text { Pregunta III-09. El fomento de la multifunción para profesores de mi unidad } \\
\text { tiene: Ventajas } 21 \% \text {, Desventajas } 27 \% \text {, Ambas } 32 \% \text {, Otro } 4 \% \text {, Sin respuesta } \\
17 \% \text {. Indicador: NC. }\end{array}$ \\
\hline $\begin{array}{l}\text { Idea sobre las labores } \\
\text { minimas de un profesor }\end{array}$ & $\begin{array}{l}\text { Pregunta IV-21. Las labores mínimas que debe cubrir la remuneración al } \\
\text { profesorado son_: Docencia e investigación, } 42.5 \% \text {; Solo docencia, } 15.9 \% \text {; } \\
\text { Docencia-investigación-extensión, } 15.2 \% \text {; Sin respuesta o no entienden, } \\
11.8 \% \text {; Inconexa, } 8.1 \% \text {; Otra, } 6.5 \% \text {. Indicador: CM. }\end{array}$ \\
\hline
\end{tabular}

Así, la mayoría coincide en que las labores mínimas justas por el salario base son la investigación y/o la docencia, pero no la multifunción; los contrasentidos afloran con la opinión mayoritaria de que la multifuncionalidad es deseable, pero no para su lugar de trabajo, y que no es el mejor estadio para fomentar los aprendizajes. La tensión se incrementa entre quienes declaran que los PEE les desestimula la práctica docente.

La reflexión sobre tales respuestas conduce a varias conjeturas a explorar a mayor profundidad en la etapa de entrevistas, para establecer la racionalidad aplicada; de momento hay evidencia de que la multifunción no es un valor firmemente instituido en este grupo y que su introyección conduce a un cambio que resulta ajeno a la mayoría. 
Otros efectos reveladores:

Por lo que se comprueba instituido del mercado capitalista, se aprecia la vinculación con el aparato productivo, la satisfacción con el significado de calidad docente y de la investigación, la necesidad de integrarse a un cuerpo académico, el contenido de la modernización, lo legítimo de competir por niveles, el apoyo a su compromiso con la investigación y conformidad con el tipo de estímulo económico.

La coincidencia se redujo en la participación de la universidad pública en la globalización, la filosofía de desarrollo humano de los PEE y la equidad remunerativa que ofrecen.

Los desacuerdos abiertos surgieron paradójicamente en que la universidad pública sea guiada por criterios capitalistas, la idea de tener que certificarse en el extranjero y en que los PEE eleven efectivamente "la productividad académica". El cruce de estas respuestas hace aflorar nuevas contradicciones susceptibles de investigación según el interés del enfoque del analista en turno.

En las nociones exploradas sobre lo instituido del Estado burocrático hay menos coincidencias: que la universidad pública debe formar científicos y profesionales, la educación por competencias y procurar democratizar el lugar de trabajo. Las coincidencias relativas se agolparon en: indecisión al pensar si la existencia de los PEE es permanente, su intención de fondo, la utilización del dinero público para estimularles, lo adecuado del sistema de constancias, la utilidad de los cuerpos académicos, la centralización de las decisiones sobre criterios, el avance en la cultura democrática, lo adecuado de la carga docente, lo correcto de acostumbrarse a ellos, la motivación a participar, lo que miden en realidad, las labores acotadas como mínimas a realizar y las mejoras académicas que se logran bajo su auspicio. No hay coincidencia respecto a quién debe fijar los criterios para la evaluación, los estímulos y los contenidos educativos, el ajuste a la autonomía universitaria, la planeación y la forma de asignar los recursos.

En las nociones de la institución democracia sí existe tendencia al acuerdo, en particular: sobre lo ideal de jerarquizar al profesorado, que el estímulo sea en dinero y prestigio, que participar en los PEE es alcanzable para todos y que estimulan a los mejores, que complementan bien el ingreso, dan tranquilidad para el desempeño de la investigación y en seguir las reglas. La coincidencia relativa fue en el deseo de volver al sistema de remuneración sin los PEE y si promueven el bienestar o los principios colectivistas. Los desacuerdos afloran en el tipo de clima laboral que hacen emerger, la preservación de la opacidad evaluativa, el tipo de ética que desatan, ciertas incomodidades al "bien ser" y las causas que dificultan la permanencia en los PEE.

Considerando que se trata de las respuestas del grupo más beneficiado en el país con los PEE, adquieren especial significado. Se trata de académicos muy competitivos, con vocación, que no consideran necesario de ser tratados bajo el principio de desconfianza, pero lo asumen.

El siguiente análisis puede ser por encontrar las coincidencias o las contradicciones más intensas que interesa explorar cómo las hacen consistentes y, de ahí, diagnosticar en conjunto un grado de institucionalización o las distancias entre el Patrón Institucional, lo débil o fuertemente instituido o el patrón en proceso de institucionalización.

\section{Conclusiones parciales}

Sabido que la sociedad es mucho más que la suma de sus partes, pero también que en la base de los procesos institucionales están las cadenas de micro-actuaciones, aquí se ha cumplido la intención de dar un orden a la dispersión conceptual y metodológica que conduzca al conocimiento sistemático de lo instituido, primero en cada informante, luego en las tendencias grupales, organizacionales -pudiendo aspirar a las nacionales-, sobre aquello que valoran los artífices del desempeño académico en contexto.

El logro inicial ha sido fijar la base institucional común de los PEE y su traducción en un marco interpretativo que propende al conocimiento cuantitativo de las nociones o mitos que los implicados valoran, dudan o rechazan. Con ello, se pone un cimiento para que los intérpretes de las diversas áreas disciplinares sociales ofrezcan sus miradas cualitativas alternativas sobre los 
efectos que se están instituyendo en el desempeño del gremio y que modelan su trato con el conocimiento.

La información generada también ha de tener utilidad para los tomadores de decisiones, en este caso particular, de los PEE, así como para los buscadores de una teoría del valor del trabajo académico, empezando con la aproximación al parecer de sus sujetos, satisfacciones y puntos de tensión.

Cada grupo ha de arrojar resultados distintos. De lograr la aplicación del método a más casos, irán aflorando relaciones institucionales comunes, con lo cual se estaría en los albores para la formulación de generalizaciones. Aunque la fundamentación cuantitativa es útil, no lo es tanto como las perspectivas cualitativas interdisciplinarias que este método ha abierto. Las vetas investigativas transdisciplinares van desde integrar la interpretación económica, sociológica, antropológica, política, psicológica y hasta mercadológica, entre las principales, para asentar lo que ha de esperarse como tendencia pesada en la forma en que se realiza la labor académica.

Las preguntas a despejar seguro correrán por saber si en la universidad pública ¿ha de avanzar el desempeño académico convergente con las directivas de los PEE supuestas como liberalizantes? ( $\sin$ asumir que así lo son); ¿cuál es el sentido político de los efectos instituyentes encontrados para el sistema universitario? de resultar representativos del resto de académicos?, ¿hacia dónde va la prospectiva universitaria mexicana con tales incrustaciones institucionales?

Por supuesto, los autores tenemos nuestras conclusiones, pero no son relevantes para los fines presentes. La relevancia está por el lado de la riqueza informativa potencial para cada tipo de intérprete, a partir de la información extraída para integrar explicaciones del fenómeno emergido del trinomio "intervención estimulativa oficial - académicos de universidad pública encuadre del conocimiento".

Si resulta que la interpretación de los distintos expertos converge y se aproxima a la realidad que ven o prefieren los informantes, entonces podría afirmarse que el método de extracción de información es útil para prever hitos universitarios y trayectorias evolutivas esperables de sus procesos.

Según los datos presentados, poca duda cabe de lo urgente de reflexionar la continuación de la forma de aplicar el tipo de estímulo, así como las pretensiones axiales de guiar al académico a reconvertirse en trabajador multifuncional e internacional, amén de la revisión de los procedimientos burocráticos que aparecen como constante tropezadero.

En la arena teórica, la aplicación del presente método sustancia el ideario de Friedland y Alford (1991) según el cual las contradicciones institucionales de Occidente penetran la operación de sus organizaciones y procesos, a juzgar por la muestra obtenida, siendo evidencia para sostener que se requiere la revisión ampliada del contenido del Patron Institucional.

Los datos permiten sostener que en el grupo estudiado aún no hay choque institucional radical o resistencias de fondo, salvo en un $10 \%$ constante de informantes en franco desacuerdo con las nociones introyectadas por los PEE. Pero entre el $4^{\circ}$ y $1^{\text {er }}$ intersticios se requiere obtener más información para proyectar los efectos esperables del comportamiento organizacional de los profesores informantes, contando con el apoyo de las entrevistas a profundidad de los psicólogos sociales.

Con apoyo en el correcto tratamiento de las inferencias que permiten los datos arrojados por el marco interpretativo, de realizar la etapa de entrevistas, también se podría identificar nuevos sentidos y significados alternativos a las nociones exploradas, ampliando y clarificando lo esperable del fenómeno, impactante de la forma de relacionarse con el conocimiento y, en alcance, del futuro universitario.

Una ilustración más del uso multidisciplinar (agregación de enfoques), no tanto interdisciplinar (integración de enfoques), que es posible hacer emergen del método, resulta de alguna acción de intervención por parte de los gestores impulsores de los PEE, quienes con conocimiento fundamentado de la forma aquí desarrollada, podrían aprovechar las inferencias para despresurizar las tensiones detectadas en cada grupo estudiado. Lo que está en juego lo vale. 


\section{REFERENCIAS}

Apple, M.W. (2000). Teoría crítica y educación. Madrid: Miño y Dávila.

Becher, Tony (1992). "Las disciplinas y la identidad de los académicos". En Universidad Futu$r a, 4$ (10). México: UAMA

Buendía Espinosa, Angélica (2011). “Análisis institucional y educación superior. Aportes teóricos y resultados empíricos”. En: Revista Perfiles Educativos, México, 33 (134).

Comas, Oscar (2003). Movilidad académica y efectos no previstos de los estímulos económicos. El caso de la UAM. México. Libros en línea ANUIES <http://www.anuies.mx/ servicios/p_anuies/publicaciones/libros/lib72/0.html>

Comas, Oscar \& Rosalía S. Lastra (2012). "La literatura sobre los estímulos económicos al profesorado universitario mexicano y la institucionalidad que introyectan en la universidad pública”. En Iztapalapa Revista de Ciencias Sociales y Humanidades. 71, UAM, México, p.p. 52-66.

Coronilla, Raúl (2004). "Propuesta metodológica para el análisis del cambio organizacional". En Los Estudios Organizacionales en México. Cambio, Poder, Conocimiento e Identidad, pp. 93-116. México: UAM

Fresán O., Magdalena et al. (2009). "El recambio generacional y la organización universitaria". En: Revista Reencuentro, 55, agosto.

Friedland, R. y R. Alford (1991). "Introduciendo de nuevo a la sociedad: Símbolos, prácticas y contradicciones institucionales". En El Nuevo Institucionalismo en el análisis organizacional. México: FCE

Ibarra Colado, Eduardo (2005). "Origen de la empresarialización de la universidad: el pasado de la gestión de los negocios en el presente del manejo de la universidad". En Revista de la Educación Superior, vol. XXXIV, Núm. 2.

Ibarra Colado, Eduardo \& Angélica Buendía (2009). "Genealogía dela evaluación y acreditación de la educación superior en México: el caso de las universidades públicas mexicanas". En: Memorias del VI Encuentro Nacional y III Latinoamericano "La Universidad como objeto de Investigación ", Córdoba, Argentina, 12. 13. 14 de noviembre.

Lastra B., Rosalía S. (2010). Estímulos al profesorado universitario en México y sus antípodas institucionales. Ed. Libros a Cielo Abierto. ISBN 978-607-7778-21-9. México: Universidad de Guanajuato.

- (2011). Informe del proyecto de investigación PROMEP Exb-138. SEP México.

Lastra B., Rosalía \& Oscar Comas (2011). Institucionalización del Profesorado Universitario en México: Efectos de los Estímulos Económicos. España: Editorial Académica Española.

López Leyva, Santos (2007) "Evaluación institucional y factores de cambio. La percepción de los académicos de tres universidades del noroeste de México”, pp. 7-22, en Revista de Educación Superior, Vol. XXXVI, Núm. 144, octubre-diciembre 2007.

López, Rodrigo (2003). "Ética de la profesión académica en la época global”. En Ética Profesional e Identidad Institucional, pp. 245-266, México: Universidad Autónoma de Sinaloa.

March, J. \& J. Olsen (1989). El redescubrimiento de las instituciones. La base organizativa de la politica. México: Universidad Autónoma de Sinaloa - FCE.

Meyer, J. \& B. Rowan (1992). Institutional and technical sources of organizational structure: Explaining the structure of educational organizations. Organizational environments, ritual and rationality. London: Sage Publications.

Muñoz, Humberto (2007). "Consideraciones sobre la política de deshomologación salarial del trabajo académico", en Daniel Cazés et al. (Coord.) Disputas por la universidad. Cuestiones críticas para enfrentar su futuro, México, Colección Educación Superior, CIICH y UNAM. 
Ordorika, Imanol (2004). “Ajedrez político de la academia”. En La academia en jaque. Perspectivas políticas sobre la evaluación de la educación superior en México. México: CRIMUNAM/Porrúa.

Powell, W. \& P. Di Maggio (1991). El Nuevo Institucionalismo en el análisis organizacional. México: FCE.

Silva, César (2007) "Evaluación $y$ burocracia: medir igual $a$ los diferentes”, pp. 7-24, en Revista de Educación Superior, Vol. XXXVI, Núm. 143, julio-sep. 2007.

Romero, Jorge (2001). "Un estudio introductorio". En El Nuevo Institucionalismo en el Análisis Organizacional, pp. 7-29. México: FCE.

Zucker, Lynn (1991). "El papel de la institucionalización en la persistencia cultural”. En El Nuevo Institucionalismo en el Análisis Organizacional, pp. 126-153. México: FCE.

\section{SOBRE LOS AUTORES}

Rosalía Susana Lastra Barrios: Doctora en Estudios Organizacionales (UAM), Master en Economía y Política Internacional (CIDE) y Licenciatura en Relaciones Internacionales (UNAM). Reconocida por el Sistema Nacional de Investigadores, Nivel I, México. Especialista en estudios sobre universidades, autora de varias obras como: Lastra B. Rosalía y Oscar Comas R. (2011). Institucionalización del profesorado universitario en México: Efectos de los Estímulos Económicos. España: Editorial Académica Española: 978-3-8443-4080-8/ Lastra B., Rosalía S., (2010) Estímulos al profesorado universitario en México y sus antípodas institucionales. Ed. Libros a cielo abierto. ISBN 978-607-7778-21-9 / Oscar Comas y Rosalía S. Lastra B. (2011). Colección Pedagogía Iberoamericana: lenguaje, formación investigativa e integral. Capítulo 12, Programas de Estímulo económico a profesores universitarios en México: un análisis por intersticios, p.p. 209-228, Editorial REDIPE, Colombia.

Oscar Comas Rodríguez: Doctor en Educación por la Universidad Autónoma del Estado de México (UAEM) y Doctor en Ciencias (Geología) por la Universidad Autónoma de México (UNAM). Actualmente es Profesor investigador "C", de tiempo completo en la Universidad Autónoma Metropolitana (UAM). 